Conference on Human Space Exploration, Space Technology \& Applications International Forum, Albuquerque, NM, Feb. 2-6 2003.

\title{
Robots and Humans: Synergy in Planetary Exploration
}

\author{
Geoffrey A. Landis \\ NASA John H. Glenn Research Center \\ mailstop 302-1 \\ 21000 Brook Park Road \\ Cleveland, $\mathrm{OH} 44135$ \\ geoffrey.landis@grc.nasa.gov \\ (216) 433-2238
}

\begin{abstract}
How will humans and robots cooperate in future planetary exploration? Are humans and robots fundamentally separate modes of exploration, or can humans and robots work together to synergistically explore the solar system? It is proposed that humans and robots can work together in exploring the planets by use of telerobotic operation to expand the function and usefulness of human explorers, and to extend the range of human exploration to hostile environments.
\end{abstract}

\section{MARS EXPLORATION}

Today's approach to human-robotic cooperation in planetary exploration focuses on using robotic probes as precursors to human exploration.

A large portion of current NASA planetary surface exploration is focussed on Mars, and robotic probes are currently seen as necessary precursors to human exploration in the areas of learning about operation and mobility on Mars, learning about the environment of Mars, mapping the planet and selecting landing sites for human mission, demonstration of critical technology on Mars, and possibly for the manufacturing of fuel before human presence and emplacement of elements of human-support infrastructure

In essence, today's approach accepts a separation of human and robotic missions-first the robots are used to scout, and then the humans explore. This is critical-it is almost unthinkable today to consider sending humans to Mars without first mapping and learning as much as possible by simpler robotic missions. Some examples of Mars robots are shown in figures 1 and 2.

\section{Robots Assisting Humans on Mars}

Beyond the use of robots as precursors to humans, robots are envisioned as assistants to astronaut exploration while the humans are on Mars (Stoker 1996). Examples of tasks that could be done by robots to assist human exploration include reconnaissance, robotic "mules", worker robots, and robotic-driven rovers.

As an example of robotic assistance, aerial reconnaissance robots can be used to help to plan traverses and locate exploration targets. This reconnaissance can be by orbital reconnaissance satellites, or real-time local aerial vehicles-- winged or rocket-powered robots.

This is a preprint or reprint of a paper intended for presentation at a publication, this is made available be made before formal not be cited or reproduced without with the understanding that it will 


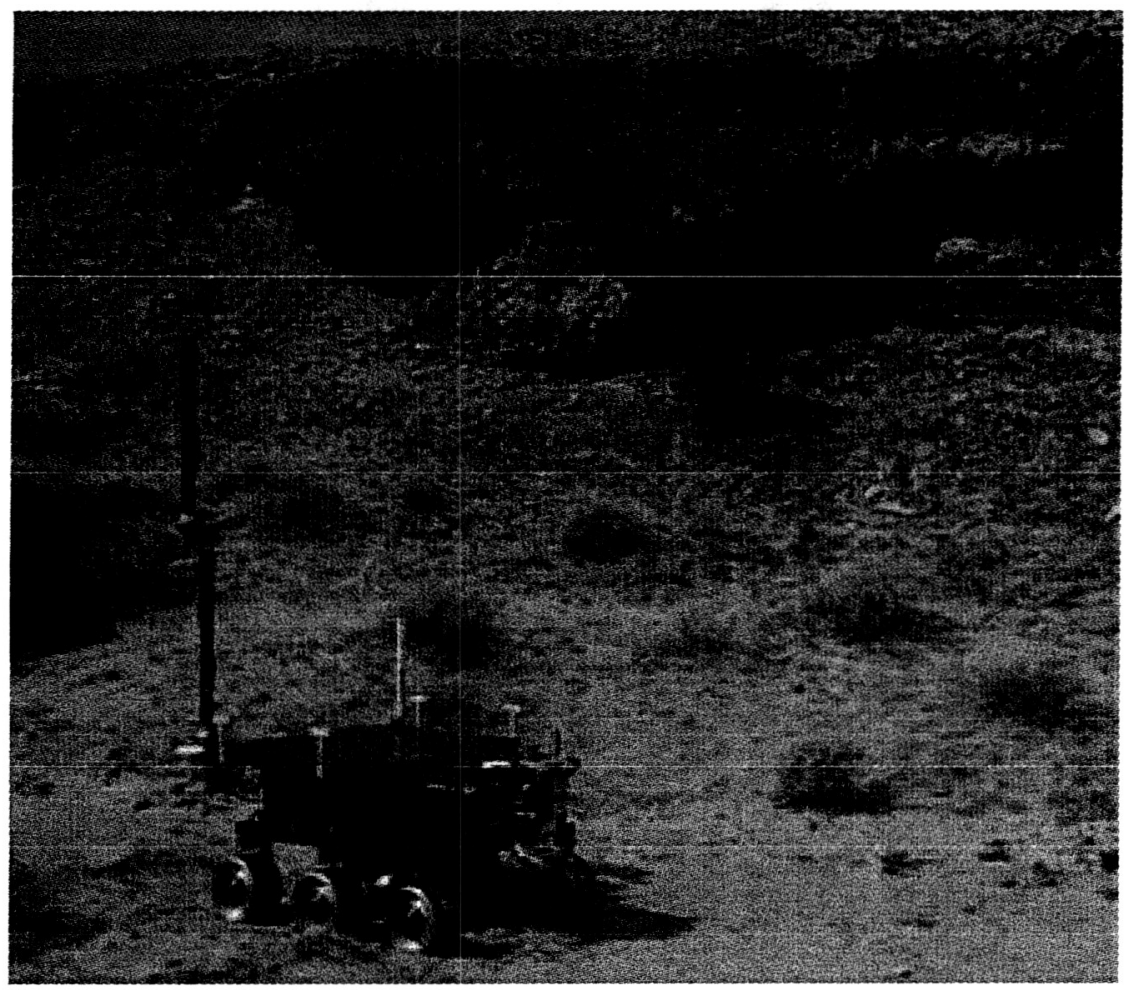

"FIDO" (Field-Integrated Design \& Operations), a prototype Mars robotic explorer, being tested in the Mojave desert (picture courtesy NASA)

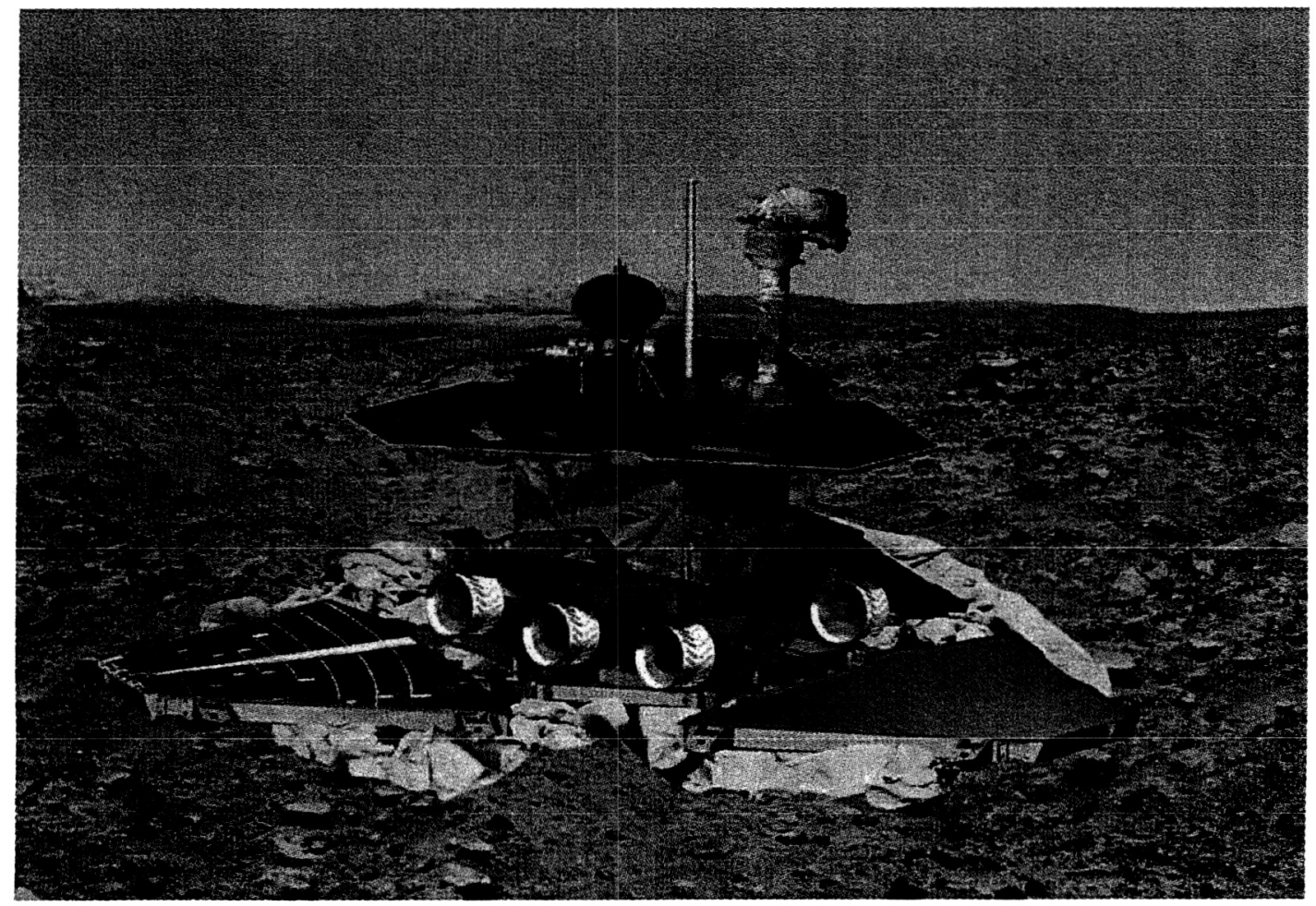

Artist's conception of the Mars Exploration rover landing by airbag on the surface of Mars in June 2004 (artist's conception courtesy NASA/Jet Propulsion Laboratory) 
One example of a robotic explorer which can assist humans in exploration by providing reconnaissance is the Mars solar-powered "scout" airplane. The Mars scout airplane is a ten-kilogram hand-launchable airplane that flies on solar power. It can carry a camera to get close-up aerial views of the local area, needed for astronauts to plan exploration and traverses, and allows real-time updating of "what's going on"... as well as giving great "outreach" views, suitable for the folks at home.

Another example is a robotic mule to follows astronauts around. The robot mule can hold tools such as rock-hammers, drills, and cameras, and will carry samples back to the habitat. It will also be ideal to aim the TV cameras for the 11 O'clock news! This removes the burden of carrying stuff from the human. Robots designed to do the grunt work of science can do tasks like setting out remote instrumentation, deploying the solar arrays and radiators, and cleaning off deposited dust after a Martian dust-storm.

Such cooperative exploration by astronauts working with robots has been demonstrated in tests by the Astronaut Robot Field Experiment ("ASRO"), discussed by Cabrol et al. (1999, 1999a).

Robotic long-range rovers take driving burden off of astronauts on long traverses. Any human landing site will be to some extent a compromise between safety and scientific interest, and many of the most interesting features may require a hundred or even thousand-kilometer traverse to reach. If the rover can be robotically driven, the astronaut effectiveness on site is increases, since the astronauts can use the travel time for rest, recreation, writing reports, answering questions from the IG travel auditor, and so forth.

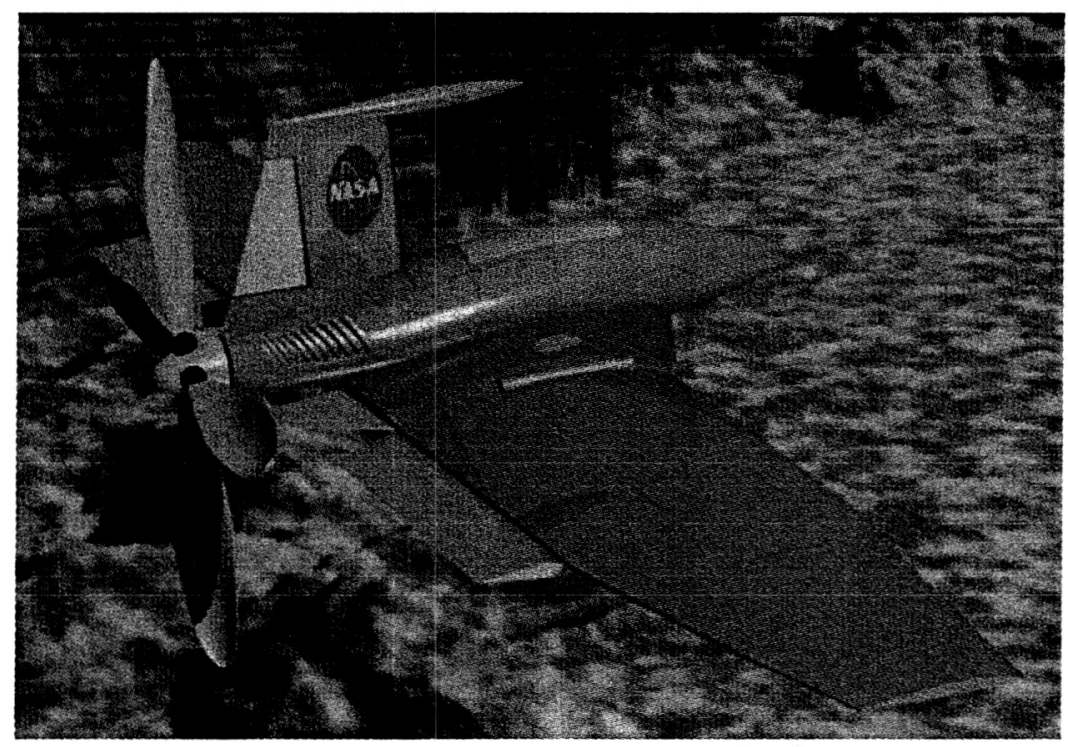

One concept for a Mars "scout" airplane. Such a vehicle could serve human exploration by providing local reconnaissance, as well as by making aerial photography of operations available (artist's conception courtesy NASA)

\section{Robots to Replace Humans for EVA}

What about robots actually replacing humans on the surface? Most roboticists make a distinction between teleoperated and remotely operated robots (Stoker 1997). A teleoperated rover has a real time operator interface (like joystick control which is routinely used for operating underwater vehicles). Often, teleoperation is assumed to include an immersive "virtual" environment, so that the human views the scene from the robot's point of view. On Mars, true teleoperation requires humans to be close enough to the robot that the speed-of-light delay is short enough that the human can operate the rover in real time.

In the telerobotic exploration scenario, the humans remain in a habitat, and use teleoperation to rove across the surface of Mars and explore. This type of exploration will require a high-fidelity, high-bandwidth connection to give the humans a fully-detailed virtual presence in the robotic body. 

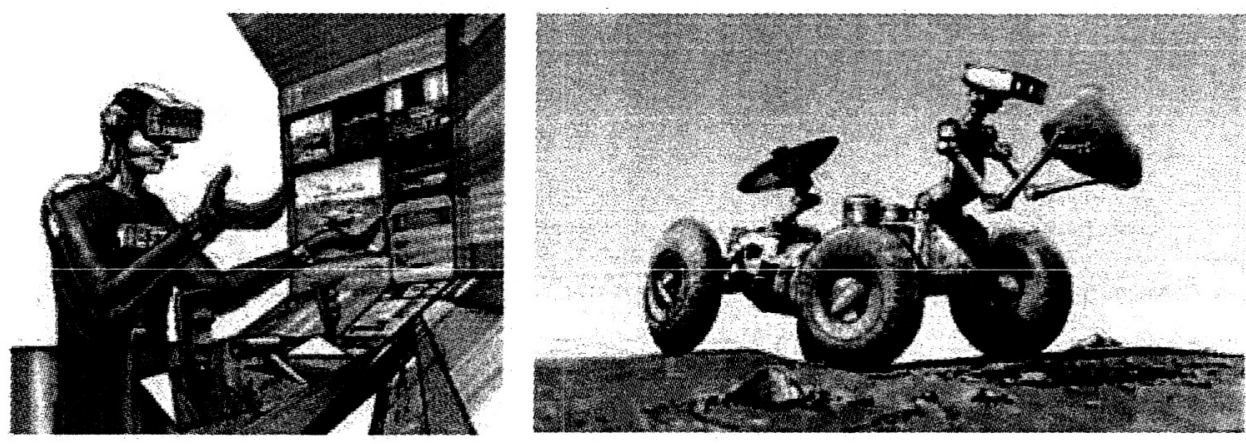

Telerobotic operations on Mars concept. [NASA Ames Research Center sketch, from Stoker (1997)].

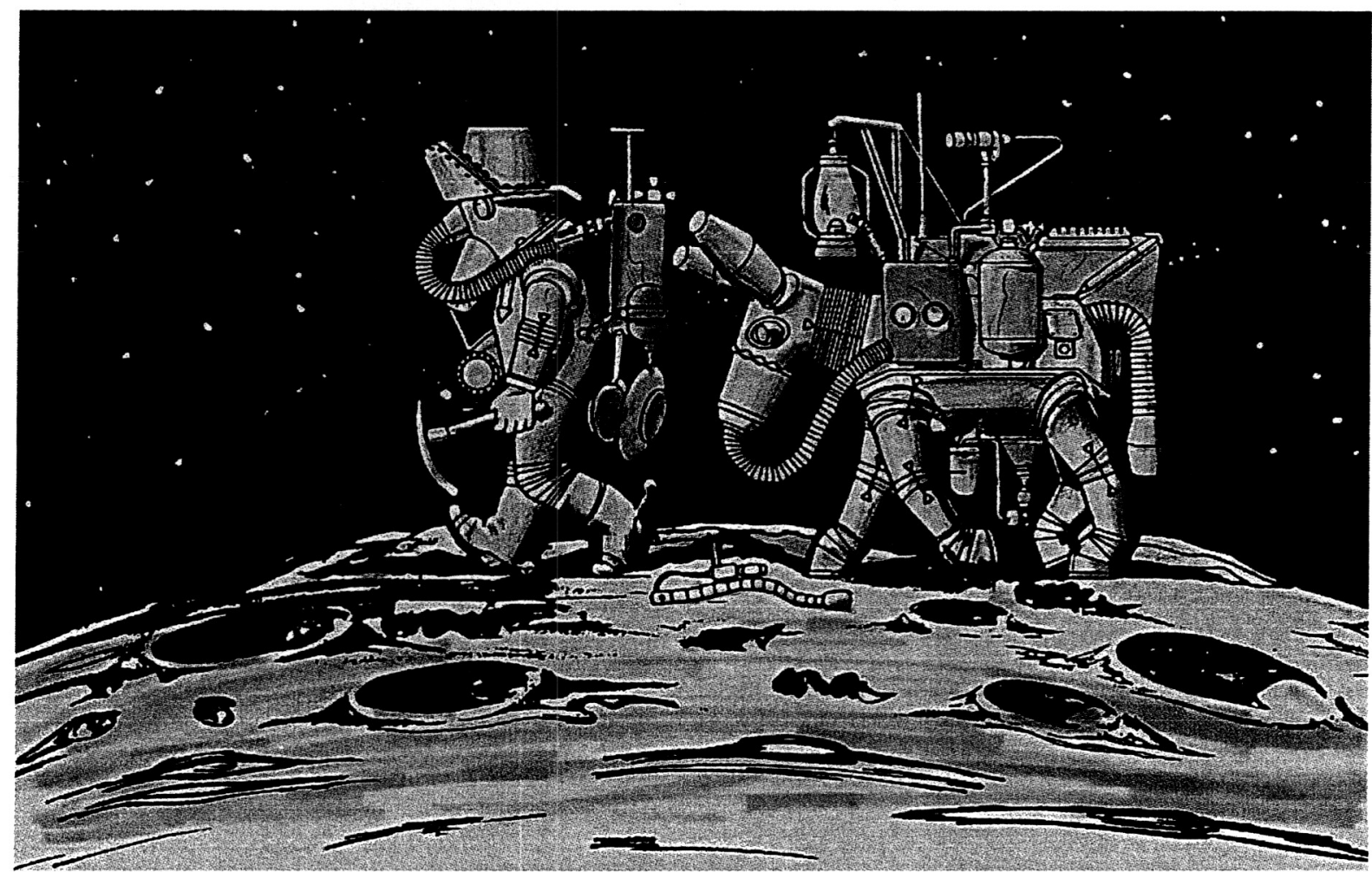

A robotic "mule" to follow astronauts with equipment could extend the capability of humans prospecting space in the same way that mules provided support for prospectors searching for gold and precious minerals in the American west (cartoon by NASA, courtesy Diane Linne)

\section{Why explore with Telerobots?}

Use of telerobots lowers risk, and thereby allows dangerous exploration. What safety committee will approve cliff-climbing in Valles Marineris? Yet the layers revealed in the canyon may very well be the key to understanding the geological history of Mars. Telerobotic exploration also allows exploration beyond walkback distance.

Use of such telerobotics will also reduce the EVA load on the astronauts. A "Marswalk" will require a time-consuming preparation, involving a lot of time expended for each hour in the field. By having the humans do their "Marswalk in a robotic body, more time can be spent on actual science.

Furthermore, robots can have expanded senses . A robot can easily have radar, infrared, and gamma-ray eyes, and so in principle a robot can see far more than a human can. 
Samples can be collected by teleoperated robots, and analyzed by humans in the shirt-sleeve environment of a fully-equipped on-site laboratory. For the most interesting sites, initial forays by telerobots can be followed by detailed "in person" visit by space-suited humans. The purpose is to save the humans for goal-oriented exploration once you know exactly where and what to look at by telerobotic exploration.

\section{A Radical Concept: Human Exploration from Mars Orbit}

Rather than land humans on the surface of Mars, we could do all of the exploration by telerobotics. What would be the result if we send humans to Mars, but don $t$ in fact land the humans on the surface? Instead, humans could explore Mars via telerobot from Martian orbit. Teleoperation of Mars surface robots from a Mars-orbital habitat will operation near real time operation with minimum time delay, giving a virtual presence on the surface (Willoughby 1988, Landis 1995).

This teleoperation vastly simplifies the mission. We now have no need to develop a human-rated Mars Lander and Mars Ascent Vehicle, and we can send geologists \& biologists on the mission; not VTOL pilots. It is a cheaper, simpler, and safer way to explore, and hence it will be a faster way to explore. Indeed, as discussed by Minsky (1990), telerobotics has at least as high human engagement factor as direct astronaut exploration: kids are excited by video games, robots, and virtual reality. It has all the excitement of being there, at a fraction of the price.

Tele-exploration from Mars orbit also allows human (virtual) presence at a wide variety of locations. With an orbital base controlling surface telerobotics, human explorers are not stuck with one base location, but can explore all over Mars. They can explore the polar caps and also near-equatorial canyon regions, from the same orbiting base. This frees the mission from landing site constraints.

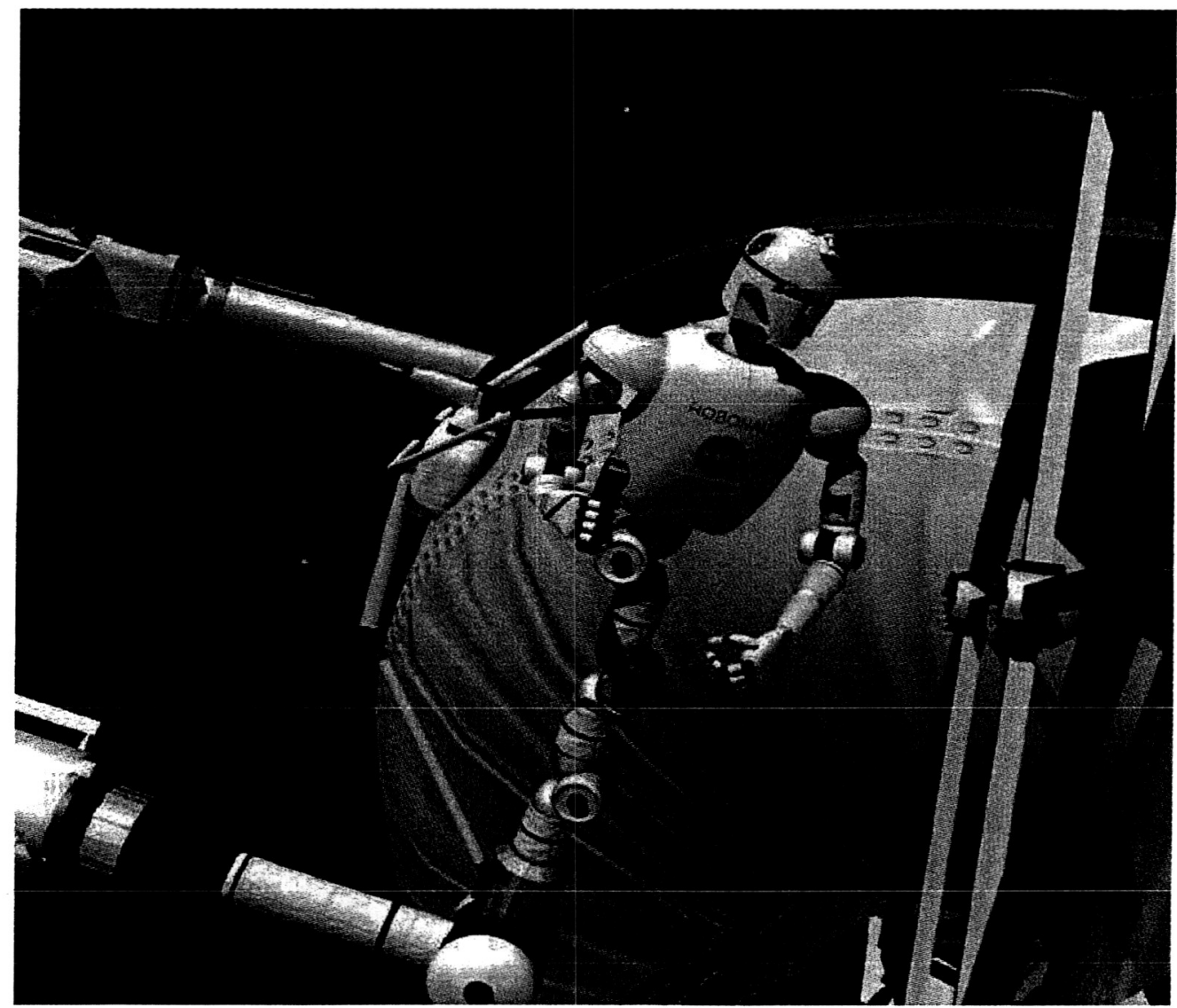

Teleoperations real-life example: "Robonaut" telerobot for EVA operation on International Space Station (Visualization by John Frassanito and Associates, courtesy NASA) 


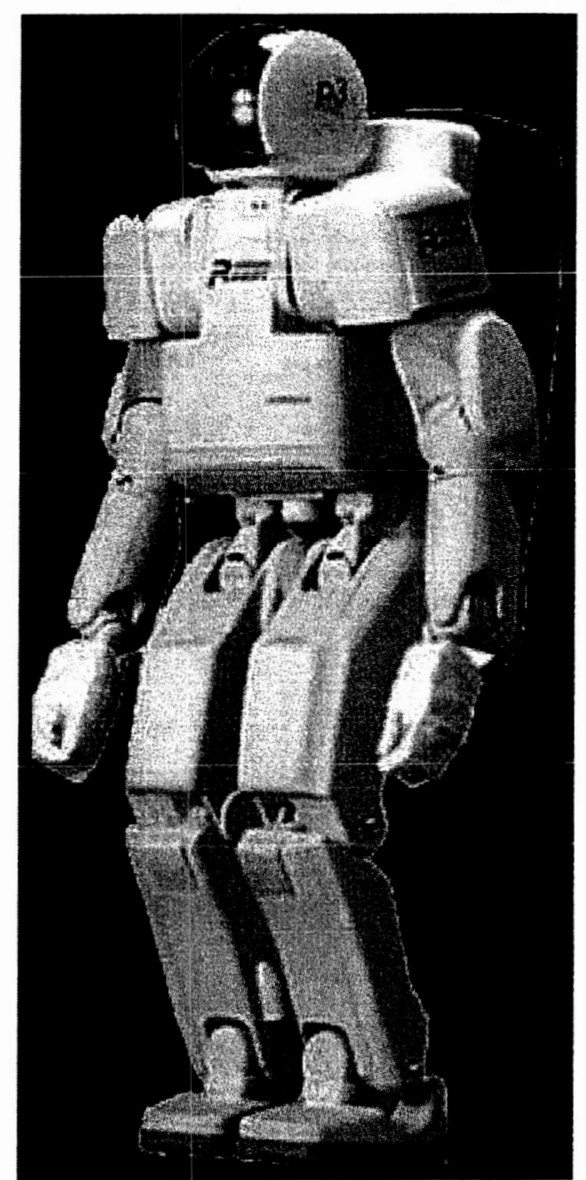

Honda Corporation unveiled their prototype "humanoid robot" in 2001. Such a robot could be a model for future telerobotic space explorers (photo courtesy Honda)

With no need to select a grab bag site that contains a large number of geologically diverse features at or near a single location; it is now possible go to all the best sites-- paleolake sites, river beds, volcanic calderas, lava tube sites, layered terrain, canyons, possible shoreline features, the North and South poles-- Mars is a huge planet, so why camp in just one place?

Near-polar exploration sites will need to be operated from a Mars-polar orbit, or an inclined orbit, to allow direct line of sight communications for surface teleoperation. A near-polar inclination 24-hr 39-minute period Mars orbit, for example, will put the orbital station in line-of-sight of a given region for about 8 hours per day-- one teleoperation shift.

\section{Why explore from Orbit? Scientific rationale}

Planetary protection is a significant (and difficult) constraint on human exploration. The most exciting question on Mars is: is there life? To answer this question, we need to explore and collect biologically pristine samples, with no terrestrial organic contamination. But all space suits are at least slightly leaky! If we explore by telerobotics, we keeps the surface of Mars sterile for biological quarantine, so when we find life, we can be sure that it is not life that we brought with us.

Since present day life could exist on Mars, planetary protection is also needed to preserve the (possible) fragile Mars biosphere from competition from ferocious Earth life. Isolated biospheres on Earth have been devastated when they have been exposed to alien life forms introduced accidentally or deliberately-- from another continent. If there is life on Mars, we will wish to protect it from having to compete with introduced Earth biota. 
Reverse planetary protection protecting the Earth biosphere from exposure to Mars microorganisms is also an important consideration. While most biologists argue that life adapted for the extreme conditions of Mars could have no possibility of infecting terrestrial life, nevertheless, from public policy alone, protecting the Earth from Mars is a primary goal. Exploring from orbit will reduce biological risk by keeping humans from exposure to possible Mars microbes. A telerobotic mission will need no quarantine on return to Earth, and avoids the difficult human question of how to isolate Mars mission astronauts infected by Martian microorganisms.

\section{BEYOND MARS: HUMAN EXPLORATION BY TELEROBOT}

\section{Exploration of Venus by Telerobot}

Venus, the greenhouse planet, is a scientifically fascinating place. In many ways it can be considered Earth $s$ evil twin. A huge number of important scientific questions remain to be answered: Before the runaway greenhouse effect, was early Venus temperate? Did Venus once have an ocean? What causes the geological resurfacing of the planet? What is the snow on Venus mountaintops? (certainly not ice!) Can we learn about Earth s climate from Venus?

The surface is far too hostile to land the humans, but we can put humans in the atmosphere to explore the surface via rugged telerobot.

The surface robot will require new technologies; specifically, it will require electronics, scientific instruments, power supplies, and mechanical linkages designed to operate at a temperature above $450 \mathrm{C}$ hot enough to melt the solder on a standard electronic circuit board. This will require devices made from advanced semiconductor materials, such as silicon carbide, or even new approaches, such as micro-vacuum tube electronics. Such materials are now being developed in the laboratory.

In the Venus atmosphere, at the one-bar pressure level, temperatures are fine for humans-- 0 to $20 \mathrm{C}$. At this level, there is plentiful solar energy. Atmospheric carbon dioxide is a plentiful resource for life support, and although humans cannot breath the atmosphere, no pressure vessel is required. Further, oxygen and nitrogen are lifting gasses on Venus: you can actually fill the envelope of an aerostat with gas you can breathe (Landis 2003).

So it should be possible to fioat in the atmosphere, and explore the surface of Venus remotely.

\section{Human Exploration of the Jupiter and Beyond by Telerobot}

Why stop at Mars and Venus? With telerobotic exploration, the whole solar system is exciting! Humans will want to explore the Jovian moons, some of the most interesting territory in the solar system. However, the moons of Jupiter orbit in an intense radiation environment. In the Jupiter system, humans will need radiation protection. Human exploration can be done with expendable radiation-tolerant robots, bringing (remote) human presence into protected habitats buried beneath the surface of one of the moons. Such an approach will expand human exploration from the sulfur volcanoes of Io to the oceans of Europa, and explore Jupiter s atmosphere by (tele-) nuclear ramjet.

Beyond Jupiter, the outer solar system is exciting, but one-way trip times of years to decades makes it unlikely to be a place for exploration within the 40 year horizon of this study. To explore the outer solar system, we need robots to send back detailed Virtual Reality models. With VR models, humans can explore in the comfort of their own planet.

Each robot sends terabits of data-- and we can send hundreds of robots. Critical to this ability to perform this mission in high-resolution virtual reality will be the ability to send high-bandwidth date by laser communications but the technology for this is here; it needs only to be adapted to space exploration.

With virtual reality, we can engage the entire population of Earth-- everybody can be an explorer! 


\section{CONCLUSION}

Today's approach to human-robotic cooperation in planetary exploration focuses on using robotic probes as precursors to human exploration, however, robots and humans can in fact be seen as systems which, when used together, can explore more effectively, and can explore a wider range of environments, than either robots or humans alone. With the use of telerobotics and virtual reality, everyone on the Earth can be an explorer.

\section{ACKNOWLEDGEMENT}

The original stimulus for this paper was the "Revolutionary Aerospace Concepts" workshop on humanrobotic exploration. It was presented in earlier form at the "Workshop on Revolutionary Aerospace Systems Concepts for Human/Robotic Exploration of the Solar System" co-sponsored by ICASE/USRA and NASA Langley, at NASA Langley Research Center, November 6-7, 2001.

\section{REFERENCES}

1. Cabrol, N. A., et al., "Results of the First Astronaut-Rover (ASRO) Field Experiment: Lessons and Directions for the Human Exploration of Mars," Fifth International Conference on Mars, July 18-23 1999, Pasadena, CA.

2. Cabrol, N. A., et al., "Astronaut-Rover Interaction for Planetary Surface Exploration: ' 99 Silver Lake first ASRO experiment." Lunar and Planetary Sciences Conference XXX, 1999, Houston TX, Abstract \#1069.

3. Landis, G. A., "Footsteps to Mars: an Incremental Approach to Mars Exploration," Journal of the British Interplanetary Society, 48, 367-342 (1995). Reprinted in From Imagination to Reality: Mars Exploration Studies, edited by R. Zubrin, pp. 339-350. AAS Science and Technology Series Volume 91, 1997.

4. Landis, G. A., "Colonization of Venus," Conference on Human Space Exploration, Space Technology \& Applications International Forum, Albuquerque, NM, Feb. 2-6 2003 (American Physical Society Conference Proceedings series; this volume).

5. Minsky, M., "Proposal for a Remotely Manned Space Station," in Vision-21: Space Travel for the Next Millennium, edited by G. Landis, NASA Conference Publication 10059 (1990), pp. 58-67.

6. Stoker, C. R., "Scientists on Mars: Science Strategy for Human Exploration," in Strategies for Mars: A Guide to Human Exploration, edited by C. Stoker and C. Emmart, AAS Science and Tech. Series 86, pp. 537-560, 1996.

7. Stoker, C., Telerobotics Course: Lecture 1, Remote Science Applications of Robots on Mars. University of North Dakota/NASA Ames Research Center, 22 January 1997. http://quest.arc.nasa.gov/courses/telerobotics/590/lecture 1.

8. Willoughby, A. J., "Multinational Exploration of Mars: An Affordable Concept", Paper IAF-88-390, 39th Congress of the IAF, October 1988. 